Letter

\title{
Anti-Wrinkle Efficacy of Neuropeptide Substance P-Based Hydrogel in Human Volunteers
}

\author{
Da Jung Kim *, Seul Bi Lee, Song Sun Chang and Jungsun Lee
}

Research and Development Institute, Biosolution, Seoul Technopark, 232 Gongneung-ro, Nowon-gu, Seoul 01811, Korea; xkssls@kaist.ac.kr (S.B.L.); kimdj1404@kaist.ac.kr (S.S.C.); xkssls@naver.com (J.L.)

* Correspondence: kimdj1404@biosolutions.co.kr

Received: 2 April 2020; Accepted: 20 May 2020; Published: 21 May 2020

\begin{abstract}
This letter is intended to clarify the clinical effectiveness of neuropeptide substance $P$ (SP) in reducing age-related skin wrinkling. This is the first clinical study to evaluate the efficacy of the topical application of SP on wrinkle reduction. For the clinical study, we tested the efficacy of a stable SP formulation known as SP-based hydrogel against skin aging in study participants. We found that this SP-based hydrogel reduced skin aging based on changes observed in several cutaneous parameters.
\end{abstract}

Keywords: substance P; hydrogel; anti-wrinkle efficacy; clinical study; cosmeceutical

\section{Introduction}

Substance P (SP), a small-sized neuropeptide consisting of 11 amino acids, is produced in wound areas and exhibits effective skin regenerative activity [1]. The anti-aging effects of SP are attributed to its anti-inflammatory activity and effects on collagen synthesis. Studies have shown that SP increases type I procollagen levels, correlated with the regulation of matrix metalloproteinase (MMP)-1 as well as anti-inflammatory activity, thus indicating its potential anti-aging efficacy in clinical practice $[1,2]$. Although the potential anti-aging activity of SP facilitates its use in cosmeceuticals, its application has been limited owing to its low stability [3]. In a previous study, a stable SP formulation known as SP-based hydrogel was developed [3]. To prevent oxidation of SP and minimize physical damage to SP from an interfacial interaction caused by SP-surfactant association and/or coating interfaces, sodium thiosulfate [4] and polysorbate 80 [5-7] were added to the SP-based hydrogel. In addition, a gelling agent (hydroxyethyl cellulose) was used to enhance the viscosity and SP stability of the hydrogel [8]. The SP-based hydrogel demonstrated superior in vitro stability and anti-aging efficacy compared to SP alone [2,3]. In particular, the SP-based hydrogel was stable in growth medium containing fetal bovine serum for up to $24 \mathrm{~h}$ and at high temperature $\left(\sim 60^{\circ} \mathrm{C}\right)$ for up to 4 weeks [3]. To evaluate SP stability, we removed aliquots of samples from the growth medium or at high temperature and then diluted them with phosphate buffered saline for analysis of SP content by enzyme-linked immunosorbent assay [3]. Although previous reports on the in vitro anti-aging effects and better stability profile of SP-based hydrogel indicate the need for clinical studies screening the anti-aging effects of SP-based hydrogel, none have been performed to date.

\section{Methods}

The anti-wrinkle efficacies of SP-based hydrogel and placebo were compared in 24 women (age, $59.5 \pm 6.9$ years; one woman who discontinued the study protocol was excluded). For the purpose of the study, ampoule formulation with SP-based hydrogel $(1 \mu \mathrm{g} / \mathrm{mL} \mathrm{SP})$ or without SP-based hydrogel (placebo, containing distilled water instead of SP-based hydrogel) was prepared. The formulation comprised several ingredients including glycerine, dipropylene glycol, 1,2-Hexanediol, sodium 
hyaluronate, tromethamine, and carbomer (Table 1). The study was performed in accordance with the Guideline for Functional Cosmetics (KFDA 11-1470000-000863-01; https:/www.mfds.go.kr/brd/ m_218/view.do?seq=708; last accessed 21 May 2020), which requires the comparative evaluation of vehicles with or without active ingredients in at least 20 patients for statistical analysis. Subsequently, 23 women were randomized to receive either topical SP-based hydrogel on the right or left side of the face or placebo without SP-based hydrogel on the other side of the face twice daily for 8 weeks. The following photographic standards of wrinkles were studied ( 0 , none; 1 , none/mild; 2 , mild; 3 , mild/moderate; 4 , moderate; 5 , moderate/severe; 6 , severe; and 7 , very severe) at weeks 0,4 , and 8 [9] by two independent dermatologists. Moreover, skin roughness (R1), maximum roughness (R2), average roughness (R3), smoothness depth (R4), and arithmetic average roughness (R5) were measured at 8 weeks using Skin-Visiometer SV 600 (Courage \& Khazaka, Cologne, Germany) [10]. For statistical analysis, SPSS software version 19.0 (SPSS Inc., Chicago, USA) was used to determine the significance of the measured values before using the test product. After repeated measurements, Analysis of variance (ANOVA) was used as a parametric method with a Bonferroni correction. In addition, Friedman test and Mann-Whitney U test were used to compare within-group data and between-group data, respectively, and $P<0.05$ was considered significant. This study was approved by the institutional review board of the Ethics Committee of the P\&K Skin Research Centre, Seoul, Korea (approval no: P1801-115).

Table 1. Formulations of substance P (SP)-based hydrogel and placebo ampoule.

\begin{tabular}{ccc}
\hline Component & Placebo $(\% w / w)$ & SP Hydrogel $(\% w / w)$ \\
\hline Water & $75.5-80.5$ & $75.5-80.5$ \\
Glycerine & $5-6$ & $5-6$ \\
Dipropylene glycol & $8-9$ & $8-9$ \\
1,2-Hexanediol & $2-3$ & $2-3$ \\
Sodium hyaluronate & $0.2-0.5$ & $0.2-0.5$ \\
Tromethamine & $0.2-0.5$ & $0.2-0.5$ \\
Carbomer & $0.2-0.5$ & $0.2-0.5$ \\
SP-based hydrogel & - & 5 \\
(SP 20 $\mu \mathrm{g} / \mathrm{mL})$ & $2000-2500 \mathrm{cPs}$ & $2000-2500 \mathrm{cPs}$ \\
Viscosity $\left(25^{\circ} \mathrm{C}\right)$ & &
\end{tabular}

\section{Results}

We found that after 8 weeks of treatment, the average scores of wrinkle grade of the SP-based hydrogel-treated side decreased significantly when compared to those before the treatment (Figure 1b). Figure 1a illustrates the remarkable decrease in wrinkles after 8 weeks of SP-based hydrogel treatment. In addition, Visiometer analysis taken at 8 weeks to analyze the five aging parameters (R1-R5) between placebo and SP-based hydrogel revealed a statistically significant decrease in all these parameters for the SP-based hydrogel-treated side $(P$-value $<0.05)$ (Figure $1 \mathrm{c})$; however, no significant decrease was noted at 4 weeks (data not shown). Nevertheless, the above values did not reflect the values prior to placebo or SP-based hydrogel treatment. To clarify this, we analyzed the percentage of change reflected in the baseline values. The change in $R$ values after 8 weeks was calculated (Figure $1 \mathrm{~d}$ ). The change value was represented as the value converted to a percentage by subtracting the baseline values from the absolute values after placebo or SP-based hydrogel treatment. As shown in Figure 1d, the rate of change in all the parameters of the SP-based hydrogel-treated side significantly improved at 8 weeks when compared to that of the placebo. Taken together, these results demonstrate the remarkable effect of SP-based hydrogel on wrinkle reduction. 
(a)

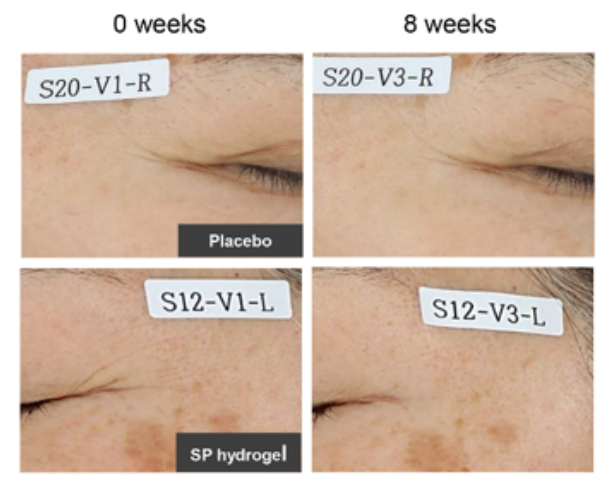

(c)

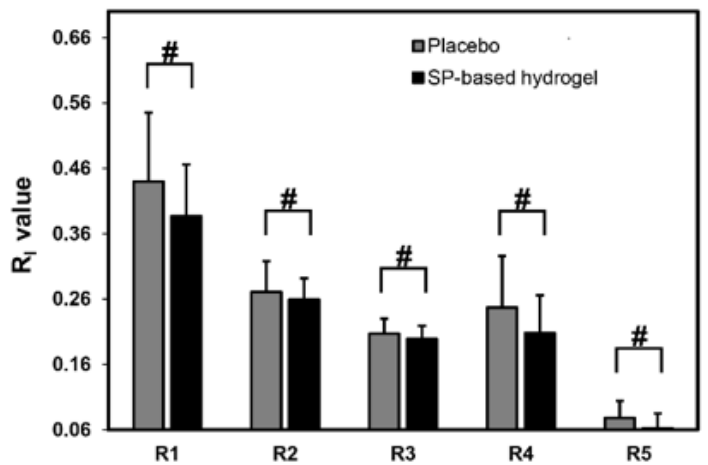

(b)

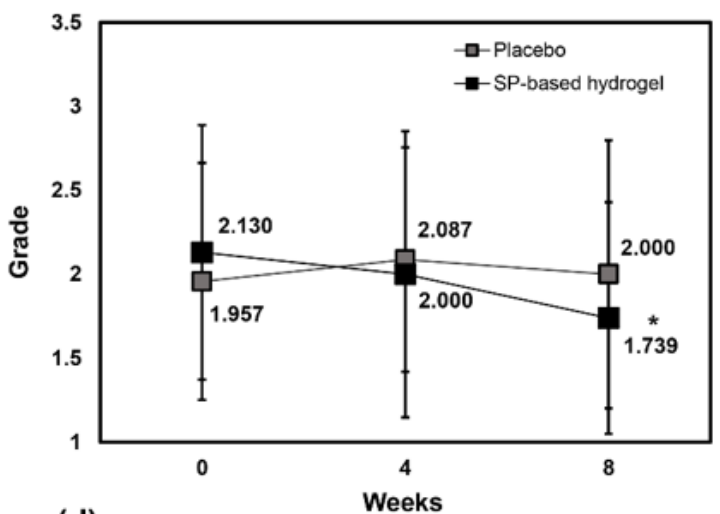

(d)

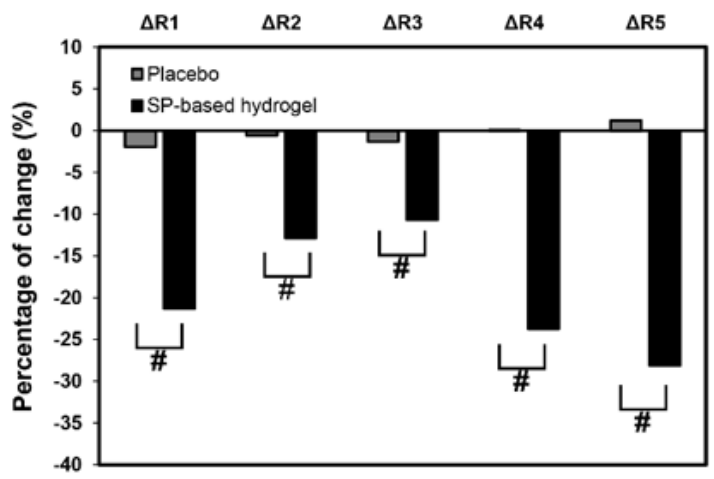

Figure 1. Changes in clinical parameters during the study: (a) participants with wrinkles at 0 and 8 weeks after treatment with placebo and substance P-based hydrogel; (b) wrinkle grade assessed by independent dermatologists at 0,4 , and 8 weeks. ${ }^{*}$ indicates a significant difference from baseline $(P$-value $<0.05$; Friedman test, post hoc Wilcoxon signed-rank test with Bonferroni correction); (c) skin replica analysis result using the Skin-Visiometer 600 after 8 weeks of treatment with placebo and SP-based hydrogel. \# indicates a significant difference from placebo ( $P$-value $<0.05$; Mann-Whitney U test); (d) changes in skin wrinkle parameters after 8 weeks. (percentage of change $=($ after - before $) /$ before $\times 100){ }^{\#}$ indicates a significant difference from placebo ( $P$-value $<0.05$; Mann-Whitney $U$ test).

To our knowledge, this is the first clinical study evaluating the efficacy of the topical application of SP on wrinkle reduction. However, further studies are required to clarify the anti-wrinkle efficacy of SP. Indeed, our study had few limitations such as (i) small sample size and recruitment of participants from a single center, (ii) lack of positive control, and (iii) short duration of screening (longer screening durations, lasting more than 8 weeks including a follow-up period are needed). To address these limitations, we are now working on a clinical study in two institutes, comparing the anti-wrinkle efficacy of SP-based hydrogel and retinol (as the positive control) for a duration of 12 weeks.

In this study, statistically significant wrinkle reduction was observed following topical application of SP-based hydrogel after 8 weeks of treatment. These findings imply that the SP-based hydrogel can be used in the cosmetic industry as a novel anti-wrinkle agent.

Author Contributions: Conceptualization, D.J.K. and J.L.; validation, S.B.L.; formal analysis, D.J.K.; investigation, D.J.K.; data curation, D.J.K.; writing—original draft preparation, D.J.K.; writing-review and editing, D.J.K.; visualization, D.J.K.; supervision, D.J.K.; project administration, D.J.K., S.S.C. and J.L. All authors have read and agreed to the published version of the manuscript.

Funding: This research received no external funding. 
Conflicts of Interest: The authors declare no conflict of interest.

\section{References}

1. Lim, J.E.; Chung, E.; Son, Y. A neuropeptide, substance P, directly induces tissue-repairing M2 like macrophages by activating the PI3K/Akt/mTOR pathway even in the presence of IFN $\gamma$. Sci. Rep. 2017, 7, 9417. [CrossRef] [PubMed]

2. Kim, D.J.; Chang, S.S.; Lee, J. Anti-aging potential of substance P-based hydrogel for human skin longevity. Int. J. Mol. Sci. 2019, 20, 4453. [CrossRef] [PubMed]

3. Kim, D.J.; Jang, J.H.; Jang, S.S.; Lee, J. A novel substance P-based hydrogel for increased wound healing efficiency. Molecules 2018, 23, 2215. [CrossRef] [PubMed]

4. Lam, X.M.; Yang, J.Y.; Celand, J.L. Antioxidants for prevention of methionine oxidation in recombinant monoclonal antibody HER 2. J. Pharm. Sci. 1997, 86, 1250-1255. [CrossRef] [PubMed]

5. Kerwin, B.A. Polysorbate 20 and 80 used in the formulation of protein biotherapeutics: Structure and degradation pathways. J. Pharm. Sci. 2008, 97, 2924-2935. [CrossRef] [PubMed]

6. Agarkhed, M.; O'Dell, C.; Hsieh, M.C.; Zhang, J.; Goldstein, J.; Srivastava, A. Effect of polysorbate 80 concentration on thermal and photostability of a monoclonal antibody. AAPS PharmSciTech 2013, 14, 1-9. [CrossRef] [PubMed]

7. Lee, H.J.; McAuley, A.; Schike, K.F.; McGuire, J. Molecular origins of surfactant-mediated stabilization of protein drugs. Adv. Drug. Deliv. Rev. 2011, 63, 1160-1171. [CrossRef] [PubMed]

8. Al-Hussein, A.; Gieseler, H. Investigation of the stabilizing effects of hydroxyethyl cellulose on LDH during freeze drying and freeze thawing cycles. Pharm. Dev. Technol. 2015, 20, 50-59. [CrossRef] [PubMed]

9. Carruthers, A.; Carruthers, J.; Hardas, B.; Kaur, M.; Goertelmeyer, R.; Jones, D.; Rzany, B.; Cohen, J.; Kerscher, M.; Flynn, T.C.; et al. A validated grading scale for crow's feet. Dermatol. Surg. 2008, 34, S173-S178. [CrossRef]

10. Kim, H.; Kim, N.; Jung, S.; Mun, J.; Kim, J.; Kim, B.; Lee, J.; Ryoo, H.; Jung, H. Improvement in skin wrinkles from the use of photostable retinyl retinoate: A randomized controlled trial. Br. J. Dermatol. 2010, 162, 497-502. [CrossRef]

(C) 2020 by the authors. Licensee MDPI, Basel, Switzerland. This article is an open access article distributed under the terms and conditions of the Creative Commons Attribution (CC BY) license (http://creativecommons.org/licenses/by/4.0/). 